\title{
Radioelectric asymmetric stimulation of tissues as treatment for post-traumatic injury symptoms
}

This article was published in the following Dove Press journal:

International Journal of General Medicine

25 August 20II

Number of times this article has been viewed

\author{
Vania Fontani ${ }^{1}$ \\ Alessandro Castagna \\ Piero Mannu' \\ Salvatore Rinaldi ${ }^{1,2}$ \\ 'Rinaldi Fontani Institute, Department \\ of Neuro Psycho Physio Pathology, \\ ${ }^{2}$ Medical School of Occupational \\ Medicine, University of Florence, \\ Florence, Italy
}

Background: For the past few years, treatment of contusions and associated symptoms, such as bruising, pain, and loss of function, has involved instrument-based therapies, ie, lasers, electromagnetic fields, and electrical stimulation. In this study, tissue optimization (TO) sessions were applied using a radioelectric asymmetric conveyor (REAC) for the treatment of contusions and associated symptoms.

Methods: Six subjects were treated with 12-18 sessions of REAC-TO applied to a traumatized anatomical area.

Results: In all cases, REAC-TO sessions were found to be effective for the treatment of posttraumatic injuries involving hematomas, hemorrhagic suffusion, and loss of function. In addition, REAC-TO treatments represented a rapid-acting analgesic associated with antiedematous, anti-inflammatory, and regenerative effects. These findings are consistent with the results of previous studies, and confirm the capacity of REAC-TO to provide almost immediate recovery of function in traumatized areas.

Conclusion: As previously demonstrated in vitro, REAC-TO is able to promote regeneration and repair processes in the human body. For six subjects experiencing local trauma, REAC-TO was effective in speeding the healing time of contusion symptoms and providing recovery of function.

Keywords: radioelectric asymmetric therapy, joint injury, muscle injury, pain, stiffness, hematoma

\section{Introduction}

For the past few years, treatment for contusions and associated symptoms, such as hematomas, pain, and loss of function, have used instrument-based therapies. ${ }^{1,2}$ These have involved the use of lasers, electromagnetic fields, and electrical stimulation.

During the acute phase of injury, bruising and pain are typically treated with the application of ice, while the intermediate phase involves topical treatments, such as painkillers and heparinoids. When loss of function is involved, rest and functional rehabilitation are often initially prescribed. Upon completion of this period, treatment with physiotherapy equipment can be used to improve symptoms further, especially when residual pain and loss of function remain. In this study, application of a new technology for biostimulation is described for the treatment of post-traumatic injuries.

\section{Materials and methods}

\section{Radioelectric asymmetric conveyor}

A radioelectric asymmetric conveyor (REAC, Convogliatore di Radianza Modulante, Italy) represents an innovative biostimulation technology creating a radiofrequency 
field that can be applied to the body. ${ }^{3,4}$ Previously, this REAC technology has been applied to several areas of medicine, including brain stimulation, ${ }^{5-11}$ tissue stimulation, ${ }^{12}$ and neuromuscular stimulation. ${ }^{13-16}$

\section{Tissue optimization}

The REAC tissue optimization (REAC-TO) protocol consists of the application of 100 radiofrequency $2.4 \mathrm{gHz}$ bursts, 0.5 seconds in duration, associated with a specific absorption rate (of $7 \mu \mathrm{W} / \mathrm{kg}$ ). The radiofrequency bursts are spaced 4.5 seconds apart, and are applied using a special laminar aluminum probe in proximity to the skin (Figure 1). Each therapy session lasts about 10 minutes, and a REAC-TO treatment cycle generally consists of 12-18 therapy sessions. REAC-TO sessions have previously been shown to be effective in improving circulation, hydration, and tropism in the skin of the face in healthy subjects. ${ }^{12}$ In as yet unpublished research, use of REAC was shown to modulate the expression of genes and proteins involved in the differentiation of stem cells and embryonic mouse cells, and to improve tissue regeneration. ${ }^{17}$

\section{Case I}

A 70-year-old woman was treated with oral anticoagulants after an accidental fall while descending a flight of stairs. The fall resulted in a significant hematoma, with hemorrhagic suffusion present in the buttocks (Figure 2) and inguinal canal (Figure 3), resulting in significant pain in the traumatized areas. Immediately following the trauma, the subject started treatment with REAC-TO. Two sessions were performed per day, one hour apart, for six days. No other therapy was locally applied while REAC-TO treatments were being conducted. Following the first session, a rapid improvement were observed in the subject's condition, and in her level of pain. As a result, an almost full recovery was achieved within six days (Figures 4 and 5).

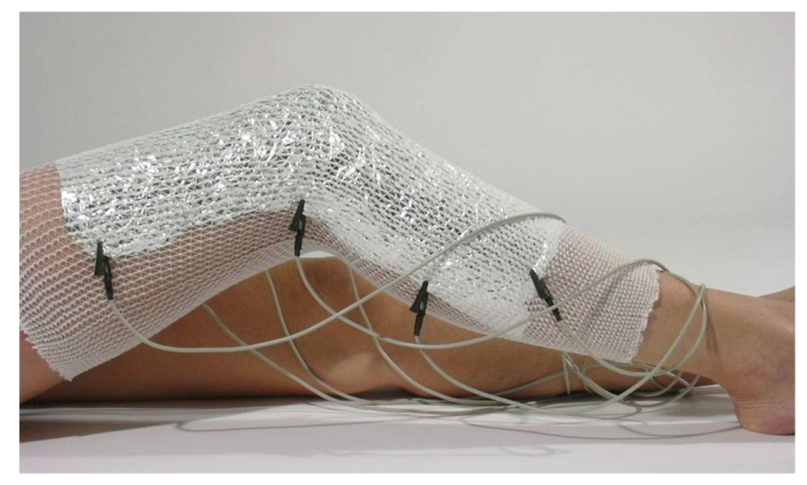

Figure I REAC Tissue Optimization (REAC-TO) protocol consisting of one hundred radiofrequency $2.4 \mathrm{GHz}$ bursts of 0.5 seconds spaced with 4.5 seconds pause, applied by a special laminar aluminum probe on the skin to treat.

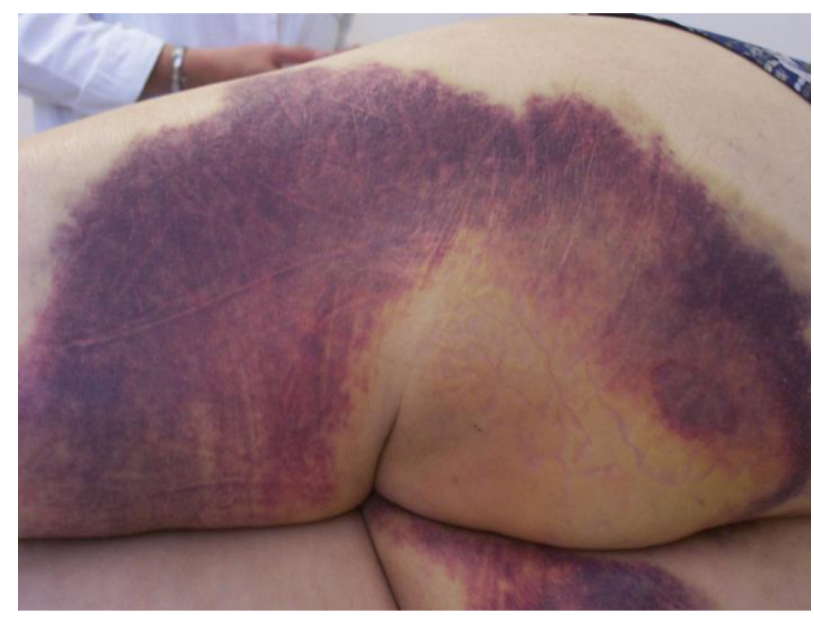

Figure 2 Female 70 years old, treated with oral anticoagulants, accidental fall while descending the stairs. Before REAC-TO treatment.

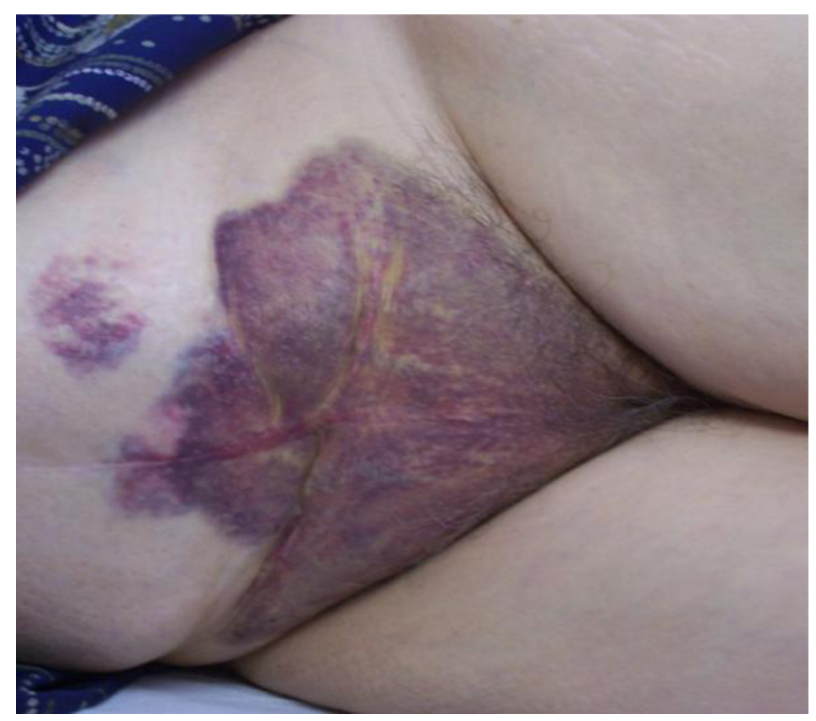

Figure 3 Female 70 years old, treated with oral anticoagulants, accidental fall while descending the stairs. Before REAC-TO treatment.

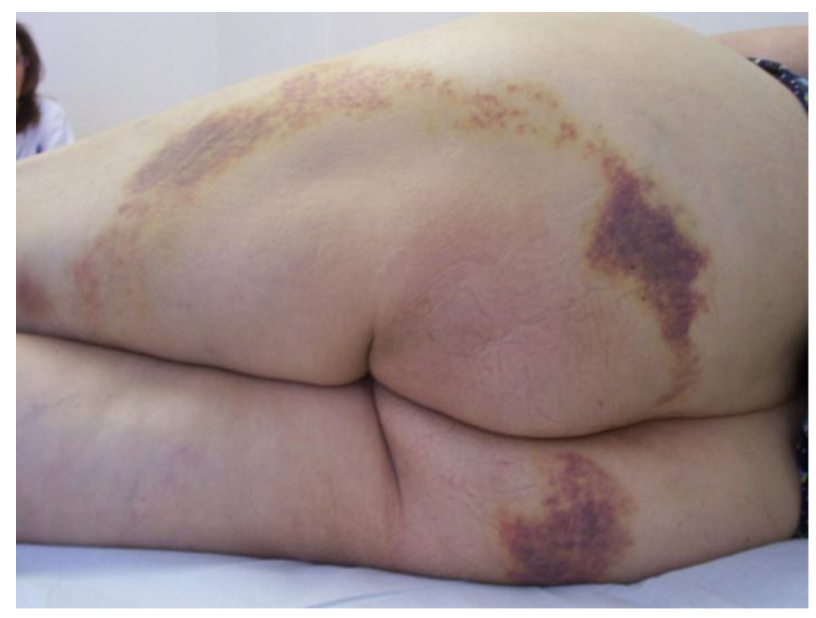

Figure 4 Female 70 years old, treated with oral anticoagulants, accidental fall while descending the stairs. After 12 REAC-TO treatment in six days. 




Figure 5 Female 70 years old, treated with oral anticoagulants, accidental fall while descending the stairs. After 12 REAC-TO treatment in six days.

\section{Case 2}

A 60-year-old man sustained an accidental trauma involving a fall on the chest, which resulted in an underarm, subfascial hematoma (Figure 6). The transverse diameter was approximately $13 \mathrm{~cm}$, and a conspicuous ecchymotic suffusion extended to the iliac wing (Figure 7). As a result of this trauma, the subject experienced severe pain when breathing and restricted arm movement ipsilateral to the lesion. Immediately following the trauma, the subject started treatment with REAC-TO. Two sessions were administered daily one hour apart on alternate days. A total of 18 sessions was conducted over three weeks. Following the first treatment, the pain experienced by the subject was sufficiently reduced to eliminate further treatment with painkillers. Furthermore, while the subject underwent REAC-TO treatments, no other therapy was administered locally. Following completion of treatment, the ecchymotic suffusion was completely resolved (Figure 8), and the hematoma present in the underarm was almost completely resolved (Figure 9). Moreover, the subject achieved full functional recovery of arm movement.

\section{Case 3}

A 30-year-old male experienced a crush injury to his right leg, with a conspicuous pretibial hematoma extending the length of the affected limb (Figures 10 and 11). Severe pain and functional loss of leg movement were reported. Treatment with REAC-TO was started two days after the injury, and included 18 consecutive daily sessions. After the first

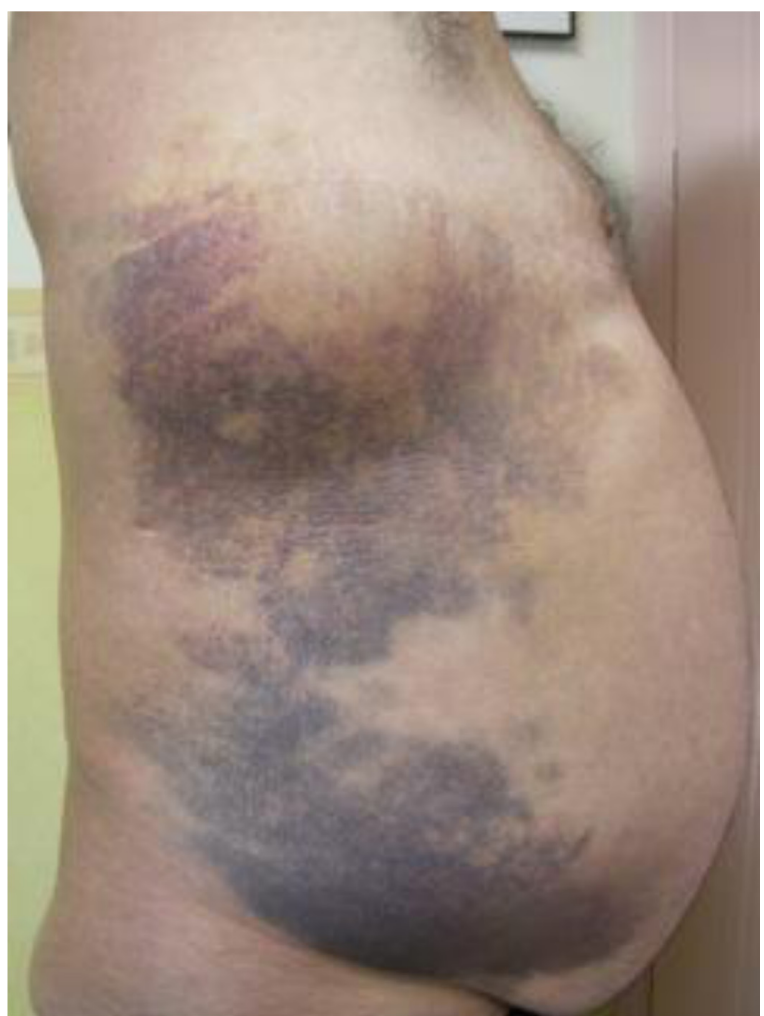

Figure 6 Male 60 years old, accidental trauma, with fall on the chest. Before REAC-TO treatment.



Figure 7 Male 60 years old, accidental trauma, with fall on the chest. Before REAC-TO treatment. 


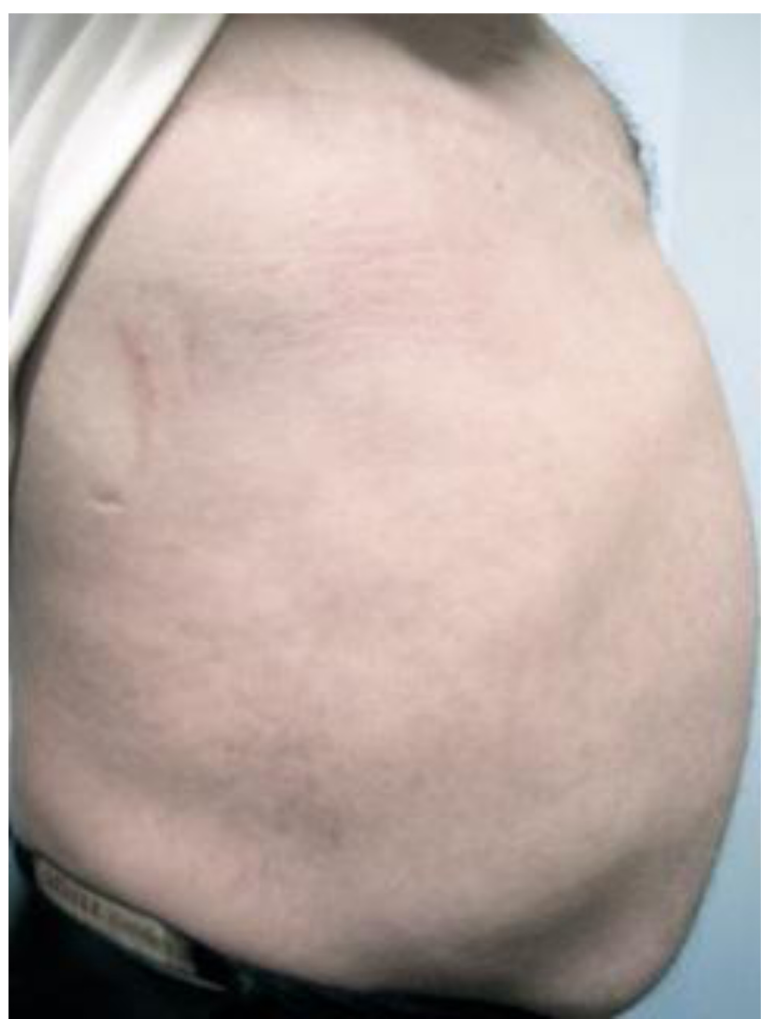

Figure 8 Male 60 years old, accidental trauma, with fall on the chest. After 18 REAC-TO treatment in three weeks.

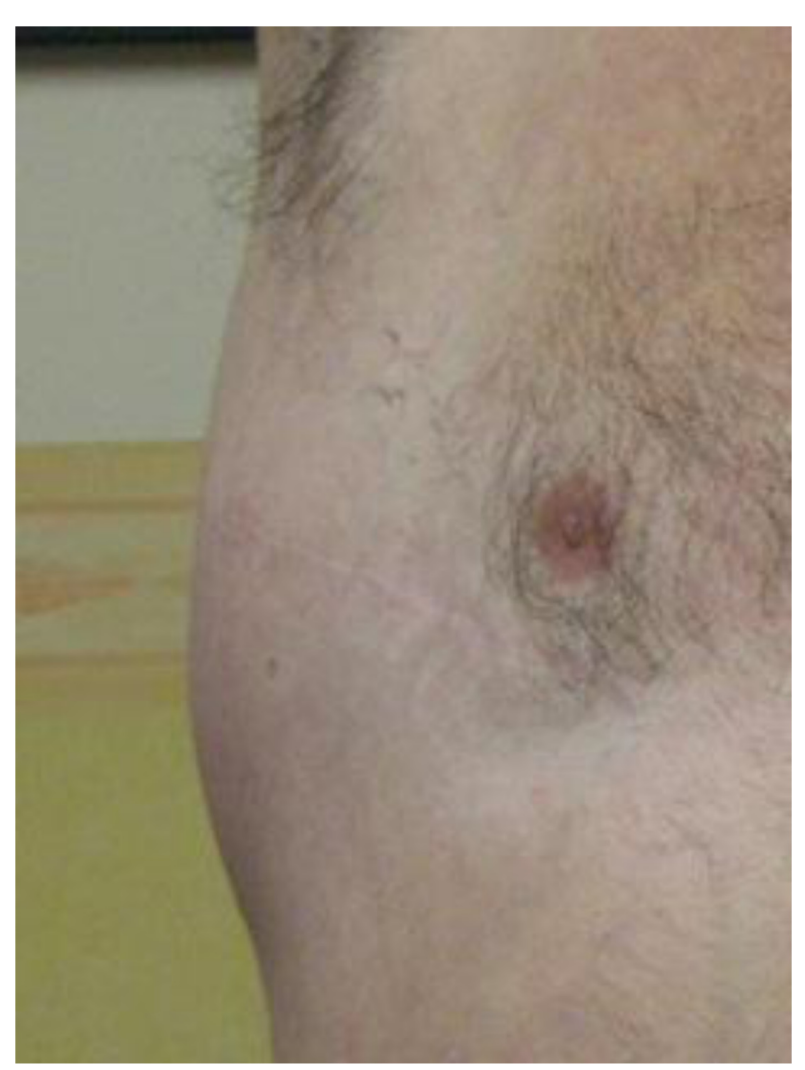

Figure 9 Male 60 years old, accidental trauma, with fall on the chest. After I8 REAC-TO treatment in three weeks.

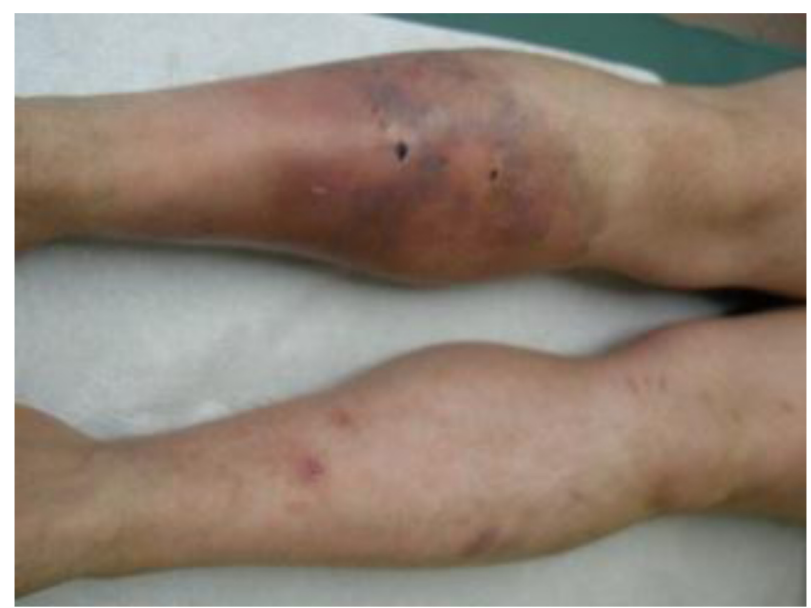

Figure 10 Male 30 years old, crushing accidental trauma on right leg. Before REACTO treatment.

treatment, a significant decrease in pain and functional recovery was reported, eliminating the need for painkillers. While REAC-TO treatments were underway, no additional local therapy was applied. Following completion of the REAC-TO treatments, modest local edema and slight discoloration from hemosiderin deposition remained (Figures 12 and 13). However, total recovery of limb function was achieved.

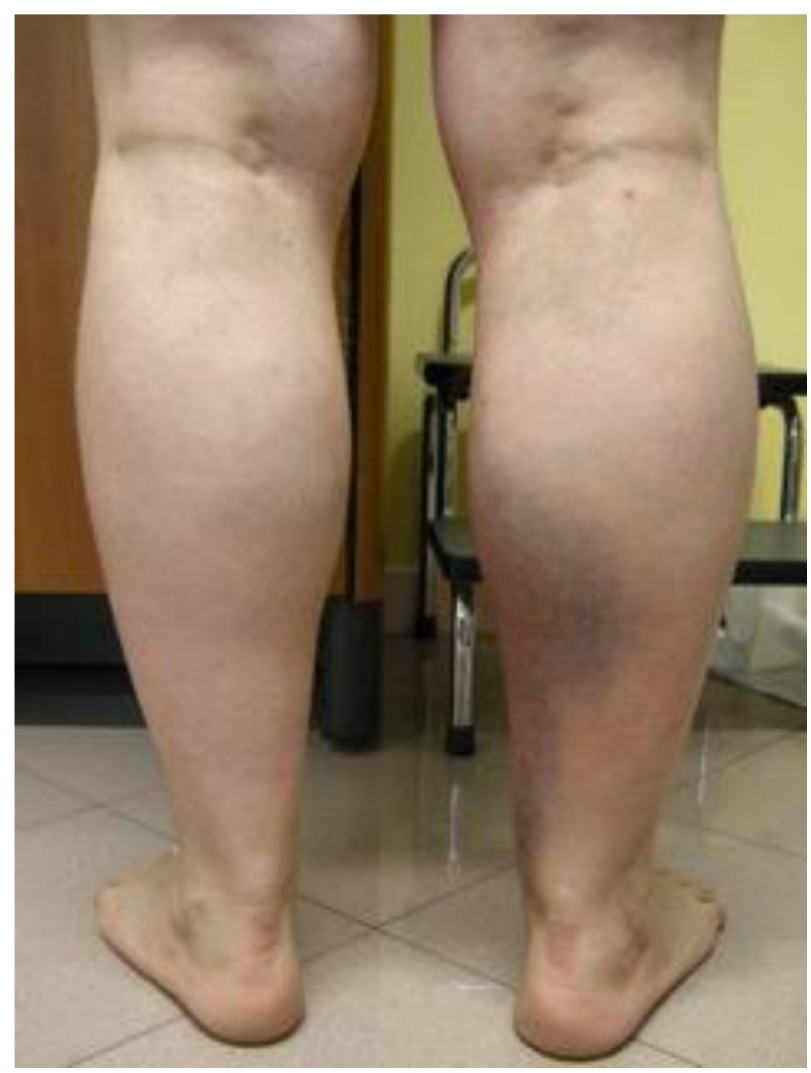

Figure II Male 30 years old, crushing accidental trauma on right leg. Before REAC-TO treatment. 


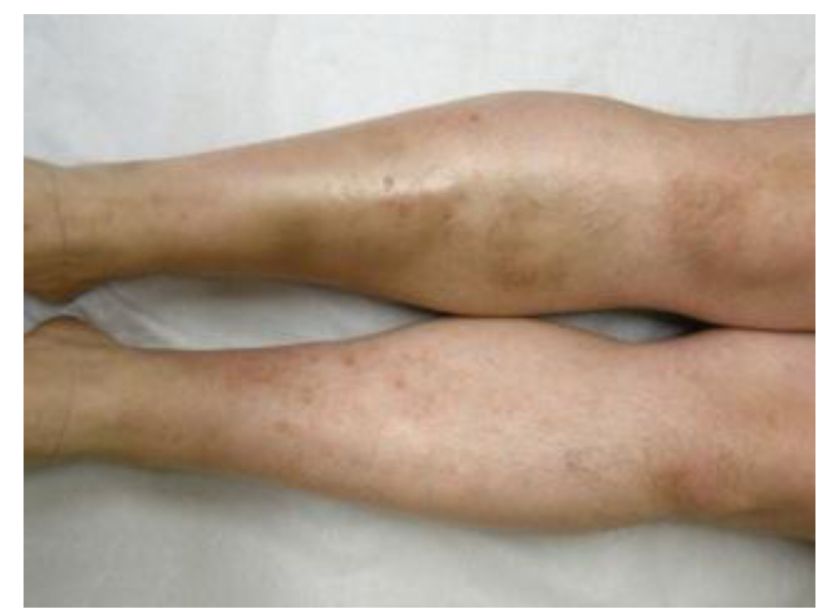

Figure 12 Male 30 years old, crushing accidental trauma on right leg. After 18 REAC-TO treatment in three weeks.

\section{Case 4}

An 18-year-old man fell accidentally on a staircase, and experienced traumatic impact to the distal third of his right leg. As a result, the subject developed a conspicuous ecchymotic suffusion that extended to the tibiotarsal joint and heel (Figures 14 and 15). Severe pain and loss of function were reported. REAC-TO treatments were started

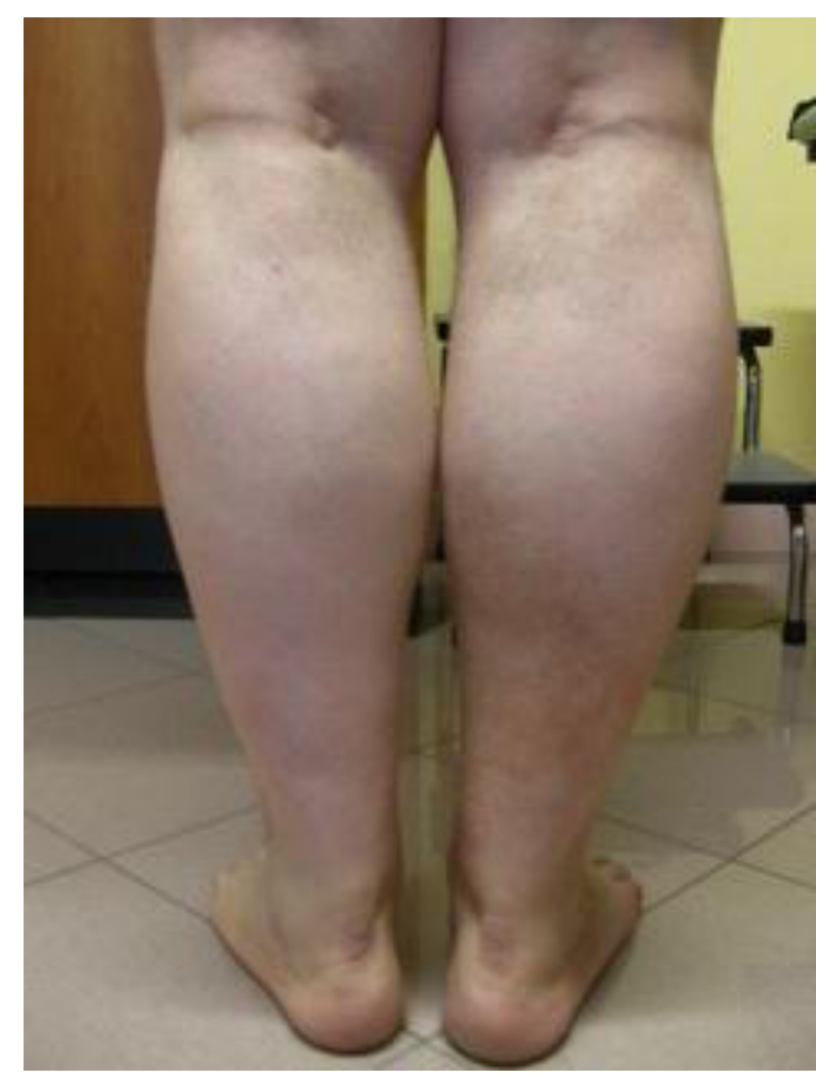

Figure 13 Male 30 years old, crushing accidental trauma on right leg. After 18 REAC-TO treatment in three weeks.

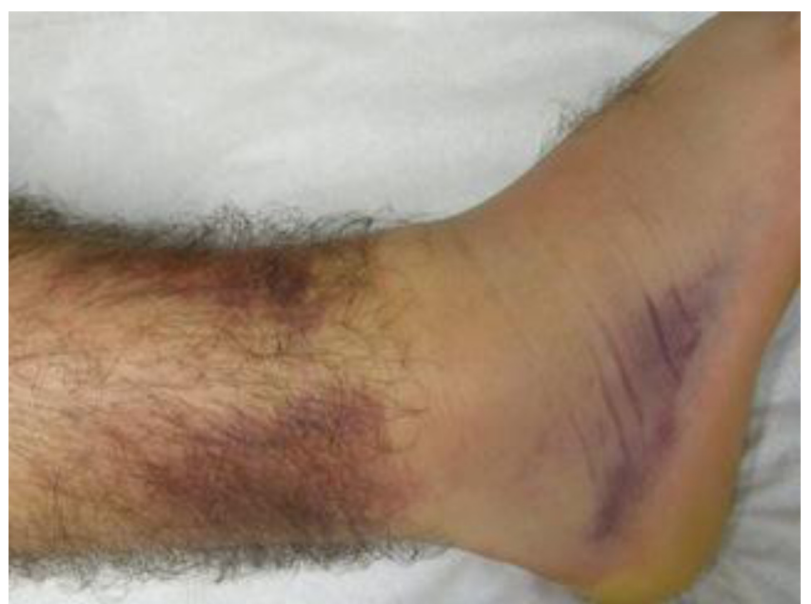

Figure 14 Male 18 years old, accidentally fell on the steps of a staircase. Before REAC-TO treatment.

immediately following the trauma, with two sessions conducted daily for 12 days. Following the first treatment, a significant reduction in pain and functional recovery were reported. During REAC-TO treatments, the subject also did not receive any other local therapies. After 12 days, complete functional recovery of the tibiotarsal joint was achieved, and the ecchymotic suffusion was resolved (Figures 16 and 17).

\section{Case 5}

A 35-year-old man accidentally fell from a ladder, resulting in a conspicuous ecchymotic suffusion that affected the tibiotarsal joint, and was associated with a hematoma in the heel (Figure 18). Severe pain and loss of function in the traumatized limb were reported by the subject. REAC-TO treatments were started immediately, with two sessions conducted daily for



Figure 15 Male 18 years old, accidentally fell on the steps of a staircase. Before REAC-TO treatment. 


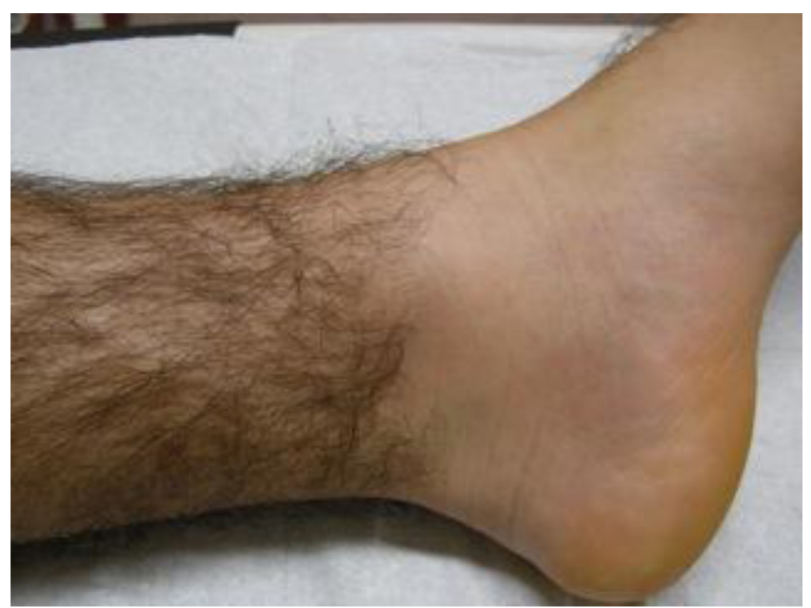

Figure 16 Male 18 years old, accidentally fell on the steps of a staircase. Images taken at follow-up done after 12 days from the end of treatment course, with 12 REAC-TO sessions performed in six days.

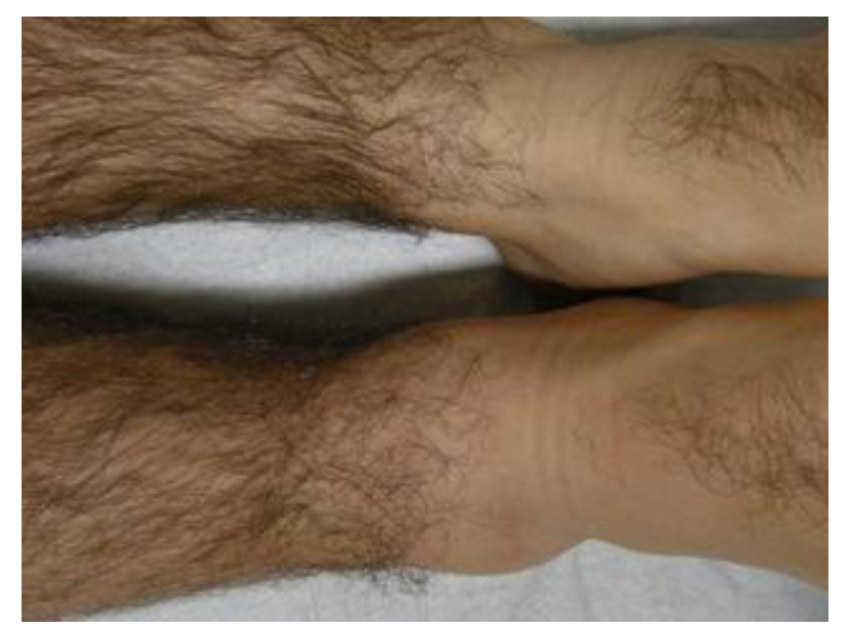

Figure 17 Male 18 years old, accidentally fell on the steps of a staircase. Images taken at follow-up done after 12 days from the end of treatment course, with 12 REAC-TO sessions performed in six days.

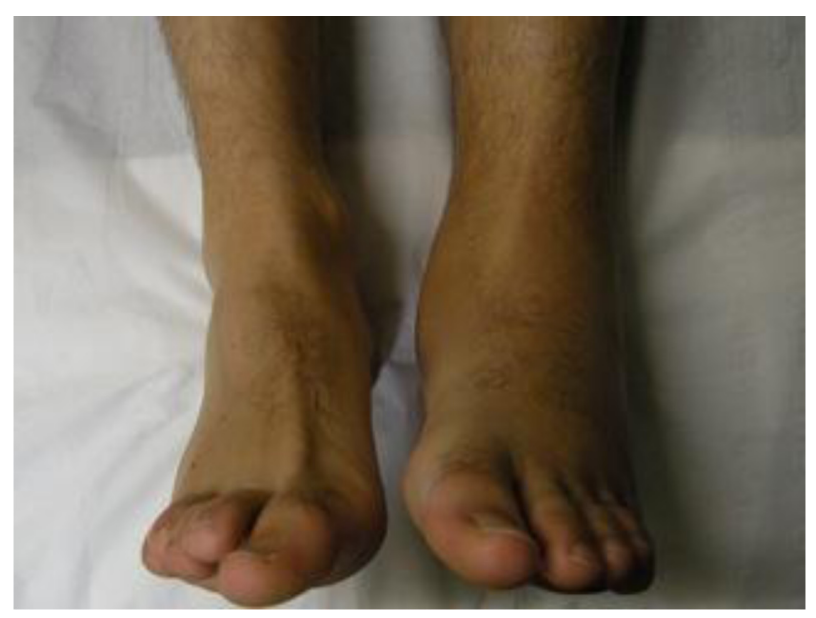

Figure 18 Male 35 years old, accidental fall from a ladder. Before REAC-TO treatment. a total of 18 sessions. Following completion of the first treatment, a significant reduction in pain was reported, and sufficient functional recovery was achieved to enable the patient to walk. The patient did not require further treatment with painkillers. Thirty days after the start of treatment, complete functional recovery of the tibiotarsal joint was demonstrated, and resolution of the hematoma in the heel was achieved. Recovery of salient bone features in the joint was also observed (Figure 19).

\section{Case 6}

A 28-year-old man was hit by a car, resulting in a conspicuous ecchymotic suffusion that extended throughout the entire leg (Figures 20 and 21). Loss of function of the leg and significant pain were reported by the subject. REAC-TO sessions were started immediately, with two sessions conducted daily for 18 sessions. Following the first session, the subject reported immediate pain relief and demonstrated early functional recovery of the leg. The subject did not require further treatment with painkillers. During REAC-TO treatments, no additional local therapy was applied. Fourteen days after the start of treatment, functional recovery of the limb was observed and complete resolution of the suffusion was achieved (Figures 22 and 23).

\section{Results}

The images presented here for subjects here clearly demonstrate the effectiveness of REAC-TO, with recovery achieved following post-traumatic injuries involving resolution of hematomas and hemorrhagic suffusion. In addition, almost immediate and progressive recovery of loss of function was observed, with REAC-TO treatments representing

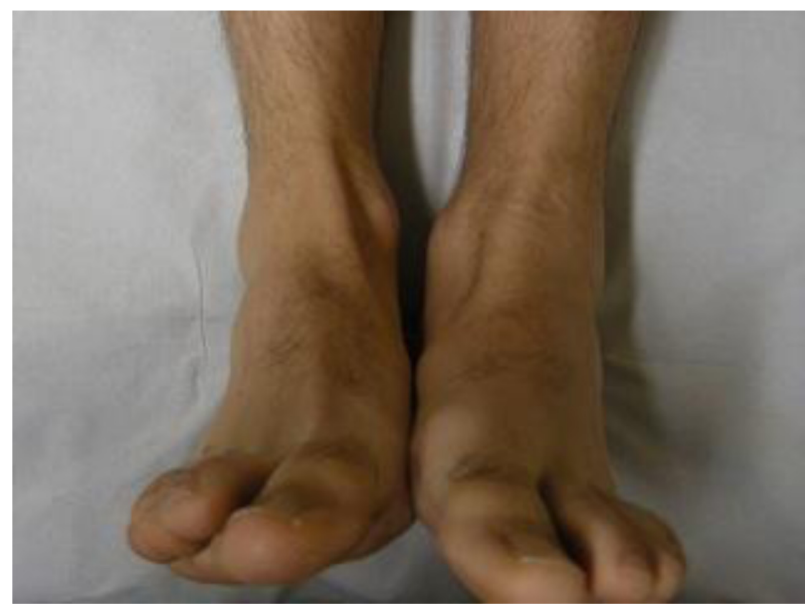

Figure 19 Male 35 years old, accidental fall from a ladder. Images taken at follow-up done after 30 days from the end of treatment course, with 12 REAC-TO sessions performed in six days. 


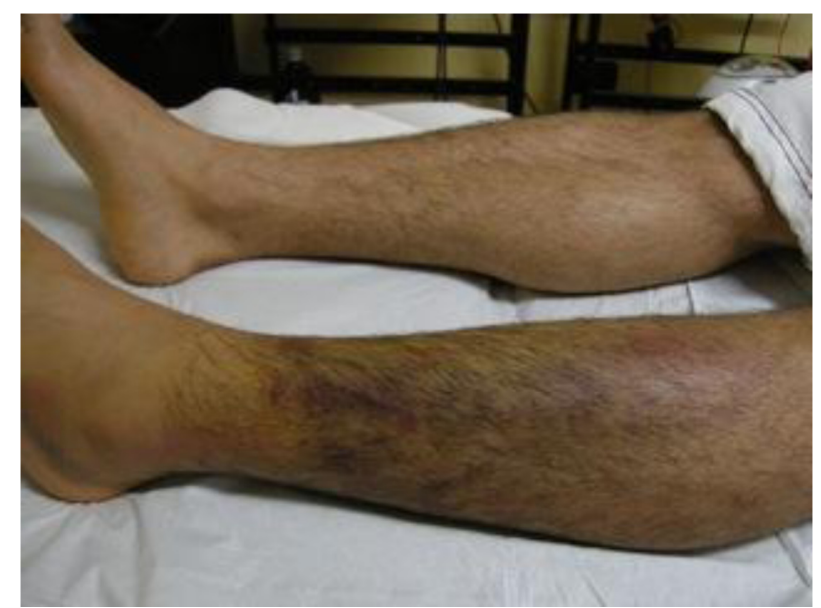

Figure 20 Male 28 years old, hit by a car. Before REAC-TO treatment.

a rapid-acting analgesic. REAC-TO treatments were also associated with antiedematous, anti-inflammatory, and regenerative ${ }^{17}$ effects.

\section{Discussion}

The cases and results presented in this study are representative of those achieved in our clinics with the application of REAC-TO to post-traumatic injuries. REAC-TO has shown

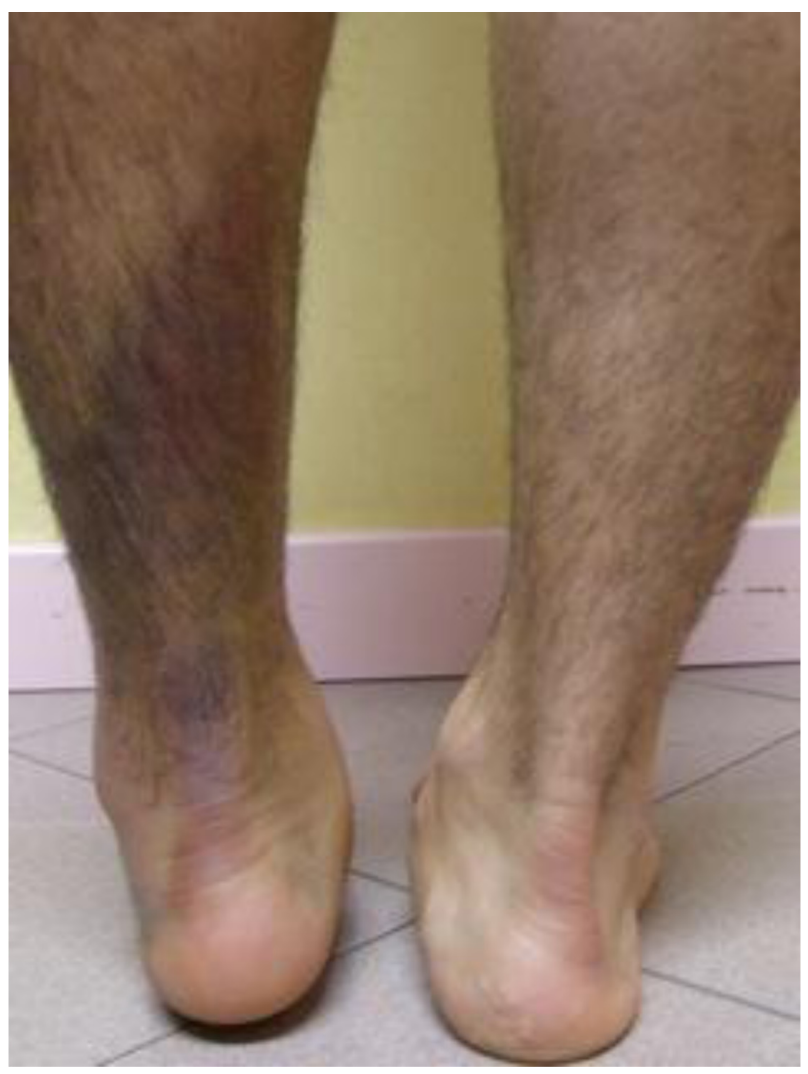

Figure 2 I Male 28 years old, hit by a car. Before REAC-TO treatment.



Figure 22 Male 28 years old, hit by a car. Images taken at follow-up done after 14 days from the end of treatment course, with 18 REAC-TO sessions performed in nine days.

efficacy in speeding the healing time of post-traumatic injuries compared with the time usually required for similar traumas treated with prompt functional recovery and rapid-acting analgesics. Furthermore, this treatment approach represents a noninvasive, painless, and safe treatment that provides functional recovery.

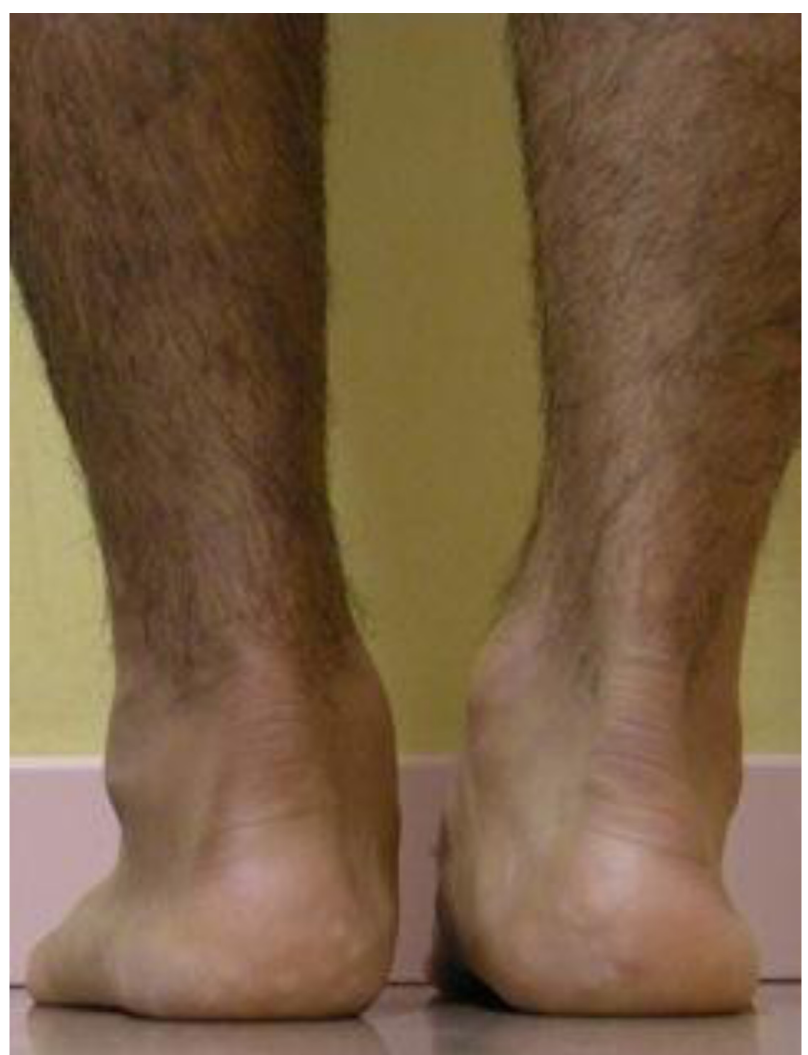

Figure 23 Male 28 years old, hit by a car. Images taken at follow-up done after 14 days from the end of treatment course, with 18 REAC-TO sessions performed in nine days. 


\section{Conclusion}

REAC-TO treatments are easy to administer, associated with rapid and effective action, especially in reducing pain and loss of function, and effective at any depth of damaged tissue. As such, REAC-TO represents a valuable tool in the treatment of various injuries, including emergency traumatic conditions.

\section{Acknowledgments}

The authors thank Dr Lucia Aravagli and Dr Stefania Bini of Rinaldi Fontani Institute, Department of Neuro Psycho Physio Pathology, Florence, Italy, for their helpful discussions.

\section{Disclosure}

$\mathrm{SR}$ and VF are the inventors of the radioelectric asymmetric conveyor.

\section{References}

1. Sheppard LA, Anaf S, Gordon J. Patient satisfaction with physiotherapy in the emergency department. Int Emerg Nurs. 2010;18: 196-202.

2. McClellan CM, Greenwood R, Benger JR. Effect of an extended scope physiotherapy service on patient satisfaction and the outcome of soft tissue injuries in an adult emergency department. Emerg Med J. 2006;23:384-387.

3. Rinaldi S, Fontani V. Radioelectric asymmetric conveyer for therapeutic use. European Patent Office: World Intellectual Property Organization. October 11, 2006.

4. Rinaldi S, Fontani V. Inventors, Rinaldi S. Fontani V, assignees. Radioelectric asymmetric conveyer for therapeutic use. US patent $7,333,8592001$

5. Collodel G, Moretti E, Fontani V, et al. Effect of emotional stress on sperm quality. Indian J Med Res. 2008;128:254-261.

6. Mannu P, Rinaldi S, Fontani V, Castagna A, Margotti LM. Radio electric treatment vs es-citalopram in the treatment of panic disorders associated with major depression: An open-label, naturalistic study. Acupunct Electrother Res. 2009;34:135-149.
7. Mannu P, Rinaldi S, Fontani V, Castagna A. Long-term treatment of bipolar disorder with a radioelectric asymmetric conveyor. Neuropsychiatr Dis Treat. 2011;7:373-379.

8. Mannu P, Rinaldi S, Fontani V, Castagna A. Radio electric asymmetric brain stimulation in the treatment of behavioral and psychiatric symptoms in Alzheimer Disease. Clin Interv Aging. 2011. In press.

9. Rinaldi S, Fontani V, Aravagli L, Margotti ML. Psychological and symptomatic stress-related disorders with radio-electric treatment: Psychometric valuation. Stress and Health. 2010;26:15323005.

10. Rinaldi S, Fontani V, Aravagli L, Mannu P. Psychometric evaluation of a radio electric auricular treatment for stress related disorders: a doubleblinded, placebo-controlled controlled pilot study. Health Qual Life Outcomes. 2010;8:31.

11. Rinaldi S, Fontani V, Moretti E, et al. A new approach on stress-related depression and anxiety: Neuro-psycho-physical-optimization with radio electric asymmetric conveyer. Indian J Med Res. 2010;132:189-194.

12. Rinaldi S, Fontani V, Cupelli V, et al. Capillaroscopy changes of the face, induced by activation with REAC tissue stimulation, in the treatment of local adaptation syndrome. Dermatologia Ambulatoriale. 2007; XV(II):29-35. (Italian)

13. Castagna A, Rinaldi S, Fontani V, Aravagli L, Mannu P, Margotti ML. Does osteoarthritis of the knee also have a psychogenic component? Psycho-emotional treatment with a radio-electric device vs intraarticular injection of sodium hyaluronate: an open-label, naturalistic study. Acupunct Electrother Res. 2010;35:1-16.

14. Castagna A, Rinaldi S, Fontani V, Mannu P. Radioelectric asymmetric brain stimulation and lingual apex repositioning in patients with atypical deglutition: a naturalistic, open-label study. J Multidiscip Healthc. 2011:4.

15. Australian New Zealand Clinical Trial Registry. Evaluation of the effectiveness of the neuro postural optimization therapy with conveyer of modulating radiance to treat functional dysmetria. 2007. Available from: http://www.anzctr.org.au/ACTRN12608000023358.aspx. Accessed on August 02, 2011.

16. Castagna A, Rinaldi S, Fontani V, Mannu P, Margotti ML. A comparison of two different treatments for coxarthrosis: local hyperthermia versus radio electric asymmetrical brain stimulation. A retrospective case series. Clin Interv Aging. 2011. In press.

17. Maioli M, Rinaldi S, Santaniello S, et al. Radio elctric asymmetric conveyed fields are able to modulate the expression of genes and proteins involved in the differentiation of embryonic mouse cells and stem cells. Paper presented at the Consorzio Interuniversitario Istituto Nazionale di Biostrutture e Biosistemi, IX Convegno Nazionale, 2010, October 21-22; Roma, Italy.
International Journal of General Medicine

\section{Publish your work in this journal}

The International Journal of General Medicine is an international, peer-reviewed open-access journal that focuses on general and internal medicine, pathogenesis, epidemiology, diagnosis, monitoring and treatment protocols. The journal is characterized by the rapid reporting of reviews, original research and clinical studies across all disease areas.

\section{Dovepress}

A key focus is the elucidation of disease processes and management protocols resulting in improved outcomes for the patient.The manuscript management system is completely online and includes a very quick and fair peer-review system. Visit http://www.dovepress.com/ testimonials.php to read real quotes from published authors. 